\title{
Central Vestibular Dysfunction in an Otorhinolaryngological Vestibular Unit: Incidence and Diagnostic Strategy
}

\author{
Badr E. Mostafa ${ }^{1}$ Ayman O. El Kahky ${ }^{1}$ Hisham M. Abdel Kader ${ }^{1}$ Michael Rizk ${ }^{1}$ \\ ${ }^{1}$ Department of ENT-HNS, Ain-Shams University, Cairo, Egypt \\ Address for correspondence Badr E. Mostafa, MD, Department of ENT- \\ Int Arch Otorhinolaryngol 2014;18:235-238. \\ HNS, Ain-Shams University, 34 higaz street 48 ibn el nafees street, \\ Cairo 11351, Egypt (e-mail: bemostafa@med.asu.edu.eg; \\ bemostafa@gmail.com).
}

\begin{abstract}
Introduction Vertigo can be due to a variety of central and peripheral causes. The relative incidence of central causes is underestimated. This may have an important impact of the patients' management and prognosis.

Objective The objective of this work is to determine the incidence of central vestibular disorders in patients presenting to a vestibular unit in a tertiary referral academic center. It also aims at determining the best strategy to increase the diagnostic yield of the patients' visit.

Methods This is a prospective observational study on 100 consecutive patients with symptoms suggestive of vestibular dysfunction. All patients completed a structured questionnaire and received bedside and vestibular examination and neuroimaging as required.

Results There were 69 women and 31 men. Their ages ranged between 28 and 73 (mean 42.48 years). Provisional videonystagmography (VNG) results were: $40 \%$ benign paroxysmal positional vertigo (BPPV), $23 \%$ suspicious of central causes, $18 \%$ undiagnosed, $15 \%$ Meniere disease, and $4 \%$ vestibular neuronitis. Patients with an unclear diagnosis or central features (41) had magnetic resonance imaging (MRI) and Doppler

Keywords

- vertigo

- dizziness

- videonystagmography

- central vestibular disorders studies. Combining data from history, VNG, and imaging studies, 23 patients (23\%) were diagnosed as having a central vestibular lesion (10 with generalized ischemia/ vertebra basilar insufficiency, 4 with multiple sclerosis, 4 with migraine vestibulopathy, 4 with phobic postural vertigo, and 1 with hyperventilation-induced nystagmus).

Conclusions Combining a careful history with clinical examination, VNG, MRI, and Doppler studies decreases the number of undiagnosed cases and increases the detection of possible central lesions.
\end{abstract}

\section{Introduction}

Vertigo and dizziness are very prevalent symptoms in daily clinical practice with a lifetime incidence of 20 to $30 \%{ }^{1,2}$ Injury to the peripheral and central vestibular system causes asymmetry in the baseline input into the vestibular centers, resulting in vertigo, nystagmus, and instability. ${ }^{3}$ Central

received

December 18, 2013

accepted

January 21, 2014

published online

March 21, 2014 causes of vertigo result from either disruption of central integrators, sensory information mismatch, or affection of the primary vestibular sensory input. Younger patients are commonly affected by migraine-associated vertigo or multiple sclerosis. Older patients are typically affected due to the associated risk factors of vascular causes of vertigo such as hypertension, atherosclerosis, and diabetes mellitus. Patients

Copyright $\odot 2014$ by Thieme Publicações License terms Ltda, Rio de Janeiro, Brazil

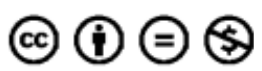


present to different specialties (otorhinolaryngologists, neurologists, general practitioners). ${ }^{2,4}$ The relative incidence of peripheral versus central disorders may differ according to the interest of the clinician, although recent publication suggests that there may be none. ${ }^{5,6}$

Diagnosis may be elusive. A thorough case history is the most important factor in determining the cause of vertigo. It provides qualitative information that can be confirmed by vestibular testing and proper radiologic studies. ${ }^{6,7}$

The primary aim of this study is to determine the incidence and nature of central vestibular dysfunction in patients presenting to a vestibular unit affiliated to an academic otorhinolaryngology department. A secondary purpose is to define a policy to improve the diagnosis of such disorders.

\section{Subjects and Methods}

This is a prospective observational study to provide actual data regarding incidence of central vestibular dysfunction in patients presenting to the vestibular unit of a tertiary referral center. The study design and observation sheet were reviewed and approved by the faculty's institutional review board.

One hundred consecutive patients with vertigo, nystagmus, nausea/vomiting, head-motion intolerance, or unsteady gait completed a structured questionnaire and received bedside and vestibular examination and neuroimaging as required.

\section{Descriptive Statistics}

A comprehensive data collection sheet was used for each patient including elements in history, clinical vestibular examination, and results of vestibular and imaging studies. Data analysis was performed utilizing Microsoft Office Excel (Microsoft Corp., Redmond, Washington, United States).

\section{Results}

There were 69 women and 31 men. Their ages ranged between 28 and 73 (mean 42.48 years). Fourteen patients had hypertension, 15 had diabetes ( 9 had both diabetes and hypertension), 5 had hyperlipidemia, 2 had severe anemia, and 1 had thyroid hypofunction.

\section{History}

Analysis of the history sheet revealed the following: 35 patients (35\%) had history (average, 3 years) of multiple attacks of rotatory vertigo lasting minutes. Of these, 19 (58\%) complained of vertigo associated with nausea and vomiting, in 14 (42\%) vertigo was initiated by body movements, and 2 (6\%) had associated neurologic symptoms (generalized weakness and/or sensory disturbances).

Another group of 31 patients complained of multiple attacks of rotatory vertigo lasting from seconds to minutes for a short duration (average 2 weeks). Within this group, 22 patients (75\%) complained of vertigo associated with hearing loss and tinnitus.
A third group of 24 patients complained of recent onset (average 2 days of episodic attacks of imbalance lasting for seconds) without any other associations.

The last group of 10 patients complained of history (average 2 years) of multiple attacks of unsteadiness lasting seconds to minutes. Six patients (56\%) complained of recurrent headaches.

\section{Clinical Vestibular Examination}

\section{Head Shake Test}

The head shake test revealed that 23 patients showed nystagmus: 13 had vertical downward beating nystagmus (56\%), 8 had vertical upward beating nystagmus (33\%), and 2 had horizontal nystagmus (11\%). The remaining patients had a negative test (77\%).

Head Impulse Test

A positive response to the head impulse test was elicited in 21 patients.

\section{Vertebral Artery Screening Test}

Three patients (3\%) had a positive vertebral artery screening test (VAST).

\section{Dix Hallpike Test}

A torsional upward beating fatigable nystagmus was detected in 31 patients (31\%). Hyperventilation induced nystagmus in 1 patient (1\%). No patients had spontaneous nystagmus. Only 1 patient (1\%) had ataxic gait. Romberg test was negative in all patients.

\section{Investigations}

\section{Audiological Assessment}

All patients underwent routine pure tone and speech discrimination audiometry. Forty-eight patients (48\%) had normal age-adjusted audiologic findings. Twenty-four (24\%) had sensorineural hearing loss of various patterns, 12 (12\%) had conductive hearing loss, and 4 (4\%) had mixed hearing loss. There was no statistical correlation between the type of hearing loss and the final suspicion of central vestibular dysfunction.

\section{Videonystagmography}

Videonystagmography (VNG) was the first screening test for all patients. Saccade abnormalities were detected in 5 patients (5\%). Smooth pursuit and optokinetic nystagmus (OKN) were normal in all patients. Positional nystagmus was found in 23 patients (23\%).

The Dix Hallpike test was positive in 40 patients. Of these, 31 patients (87\%) had torsional upward beating fatigable nystagmus, and 9 patients (13\%) had torsional downward beating nystagmus.

Caloric test showed that 5 patients had unilateral weakness, 1 patient had hyperexcitable caloric test, and 1 patient had bilateral caloric weakness. 
Provisional VNG results were $40 \%$ benign paroxysmal positional vertigo (BPPV), 23\% suspicious of central causes, $18 \%$ undiagnosed, $15 \%$ Meniere disease, and $4 \%$ vestibular neuronitis.

Although saccades, smooth pursuit, and OKN test central pathways, in our study only two tests suggested a central lesion: the positional test (90\%) and the caloric test (60\%). VNG data were insufficient to diagnose central dysfunction.

\section{Magnetic Resonance Imaging}

Patients in which a diagnosis could not be reached, those with a suspicion of central lesions, and those with risk factors (diabetes mellitus, hypertension, hyperlipidemia; 41 cases) had a magnetic resonance imaging (MRI) study (with contrast and diffusion-weighted images). Only 39 underwent the study. Eleven patients (27\%) had abnormal findings: 4 had multiple sclerosis (38\%) and 7 had generalized ischemia (62\%). The remaining had normal findings (73\%).

\section{Carotid and Vertebrobasilar Duplex}

Carotid and vertebrobasilar duplex was performed in 28 patients ( 3 patients with positive VAST and those with cardiovascular risk factors). Increased resistivity indices and significant vessel narrowing could be documented in 6 patients ( 2 in the carotid system and 4 in the posterior circulation).

\section{Incidence}

Among the 100 patients presenting with vestibular dysfunction, 23 patients had central vestibular features. Among the 23 patients,10 patients had generalized ischemia/vertebra basilar insufficiency, 4 had multiple sclerosis, 4 had probable migraine vestibulopathy, 4 had phobic postural vertigo, and 1 had hyperventilation-induced nystagmus .Among the 10 cases of generalized ischemia, 4 were previously diagnosed and VNG was performed for recurrent attacks of vertigo during follow-up. Only 2 cases presented primarily to the vestibular clinic. Two were diagnosed by suspicious history and positive VAST and carotid duplex that showed impaired flow in the vestibular system. MRI detected suggestive changes in 9 patients.

None of the 4 cases of multiple sclerosis were diagnosed beforehand. Three presented with long-standing history ( $>4$ years of recurrent attacks of rotatory vertigo lasting for minutes) with normal gait, and the only positive findings was nystagmus on head shake test (HST). The fourth patient presented with multiple attacks of vertigo and vomiting not responding to any medical treatment. He had many VNG studies with normal findings, and the MRI revealed inactive demyelinating process.

The otoneurologic examination in the 4 cases of migraine vestibulopathy was typically unremarkable. By the Neuhauser and Lempert criteria, 2 had a definitive migraine and 2 had probable migraine.

The 4 patients with phobic postural vertigo were diagnosed by International Statistical Classification of Diseases and Related Health Problems, 10th revision criteria in the department of neuropsychiatry.

\section{Discussion}

Central vestibular dysfunction is still difficult to recognize in outpatient and emergency departments. This may be due to a low suspicion index or the complex presentations. The incidence is highly variable among different centers and specialties: low figures were reported by Drachman and $\mathrm{Hart}^{8}$ (2\%) and Korres et $\mathrm{al}^{9}$ (3.9\%), and a much higher incidence was reported by Kroenke et $\mathrm{al}^{10}(11 \%)$, Delémont and Rutschman ${ }^{7}$ (15\%), Junicho et $\mathrm{al}^{11}(18 \%)$, and Karatas ${ }^{12}$ (25\%). The differences are mostly due to the diagnostic protocols and criteria set by each group. The detection of a central disorder in patients with a balance disorder is important. The incidence of subsequent cerebrovascular events in patients presenting initially with dizziness, vertigo, and instability is higher than expected. ${ }^{13-16}$ Central vertigo should be suspected and brain imaging performed in the presence of neurologic symptoms, in older patients, or when several risk factors for cerebrovascular disease are present. ${ }^{17,18}$

This study is a database to provide actual data regarding the diagnosis of patients presenting with vestibular dysfunction at a tertiary referral university department. One hundred consecutive unselected patients presenting to the vestibular unit were assessed clinically and audiologically by vestibular testing and radiologically to determine the cause of their dizziness. The data files were collected and stored electronically in a uniform file structure that allows data elements to be manipulated, correlated, or extracted to satisfy the diverse analytical and reporting needs. In this study, the incidence of patients with a final tag of central vertigo was $23 \%$. The main reasons for this high incidence may be the following. There may be a referral bias as it is a tertiary university center, with selected cases directed to it. There was also a high suspicion index maintained during the study and the follow-up of vestibular testing by other investigative modalities. In most centers during routine practice, patients are referred to undergo vestibular testing and are usually referred back without follow-up. VNG data are used to differentiate between central and peripheral disorders and for classifying peripheral disorders, but there are many pitfalls and in many cases a definitive diagnosis could not be reached. ${ }^{18,19}$ In our study, two tests suggested a central lesion-the positional test (90\%) and the caloric test (60\%)-whereas central oculographic tests were not conclusive. This is insufficient, and whenever a central pathology is suspected or a diagnosis cannot be reached, the patient should undergo further workup. ${ }^{9,11,20,21}$ In this study, MRI suggested the diagnosis in $27 \%$ of the studied patients. ${ }^{9,22,23}$

Therefore, we combined history, clinical examination, VNG, VAST carotid, vertebrobasilar Doppler, and MRI in all patients with suspected central lesions. Using our protocol, $\sim 40 \%$ of patients were properly diagnosed. We diagnosed patients with vertebrobasilar insufficiency, discrete vascular injuries, syringomyelia, and multiple sclerosis. ${ }^{12,15,24}$ On the other hand, migraine vestibulopathy had no definite reported findings in VNG or MRI and may be peripheral or central. There is no single diagnostic test specific for migraine vestibulopathy (MV). Only history and associated symptoms 
together with neurologic examination is the clue to diagnose migraine and its associated vertigo. ${ }^{25-27}$

\section{Conclusion}

The incidence of central causes for vertigo and/or instability in patients presenting to an otorhinolaryngology-affiliated vestibular unit is quite high. Diagnosis of central vestibular dysfunction is very difficult and needs a high index of suspicion, a trained physician, compliant patients, and detailed investigations. Routine VNG examination is not sufficient for diagnosis. However, in the presence of symptoms and negative tests, this should direct the physician to investigate further. The combination of VNG, Doppler, VAST, and MRI raises the diagnostic accuracy to nearly $40 \%$, and we suggest that this becomes a routine algorithm in the evaluation of all patients with vestibular disorders.

\section{References}

1 Aminoff MJ, Greenberg DA, Simon RP. Clinical Neurology. 6th ed. New York, NY: Lange Medical Books/McGraw-Hill; 2005; 113 ;

2 Strupp M, Brandt T. Diagnosis and treatment of vertigo and dizziness. Dtsch Arztebl Int 2008;105(10):173-180

3 Walker MF, Zee DS. Bedside vestibular examination. Otolaryngol Clin North Am 2000;33(3):495-506

4 Hanley K, O'Dowd T, Considine N. A systematic review of vertigo in primary care. Br J Gen Pract 2001;51(469):666-671

5 Agrup C, Gleeson M, Rudge P. The inner ear and the neurologist. J Neurol Neurosurg Psychiatry 2007;78(2):114-122

6 Strupp M. [Vertigo and dizziness: the neurologist's perspective]. Ophthalmologe 2013;110(1):7-15

7 Delémont C, Rutschmann O. [Vertigo: it all revolves around the physical exam]. Rev Med Suisse 2007;3(121):1826-1828, 18301832

8 Drachman DA, Hart CW. An approach to the dizzy patient. Neurology 1972;22(4):323-334

9 Korres S, Riga M, Papacharalampous G, et al. Relative diagnostic importance of electronystagmography and magnetic resonance imaging in vestibular disorders. J Laryngol Otol 2009;123(8): 851-856

10 Kroenke K, Hoffman RM, Einstadter D. How common are various causes of dizziness? A critical review. South Med J 2000;93(2): 160-167, quiz 168
11 Junicho M, Fushiki H, Aso S, Watanabe Y. Prognostic value of initial electronystagmography findings in idiopathic sudden sensorineural hearing loss without vertigo. Otol Neurotol 2008;29(7): 905-909

12 Karatas M. Central vertigo and dizziness: epidemiology, differential diagnosis, and common causes. Neurologist 2008;14(6): 355-364

13 Chase M, Joyce NR, Carney E, et al. ED patients with vertigo: can we identify clinical factors associated with acute stroke? Am J Emerg Med 2012;30(4):587-591

14 Lee $\mathrm{W}$, Chen L, Waterston J. Vertebrobasilar ischaemia presenting as recurrent isolated vertigo. Acta Otolaryngol 2011;131(8): 887-889

15 Della-Morte D, Rundek T. Dizziness and vertigo. Front Neurol Neurosci 2012;30:22-25

16 Lee $\mathrm{H}$. Audiovestibular loss in anterior inferior cerebellar artery territory infarction: a window to early detection? J Neurol Sci 2012;313(1-2):153-159

17 Delaney KA. Bedside diagnosis of vertigo: value of the history and neurological examination. Acad Emerg Med 2003;10(12):1388-1395

18 Cnyrim CD, Newman-Toker D, Karch C, Brandt T, Strupp M. Bedside differentiation of vestibular neuritis from central "vestibular pseudoneuritis.". J Neurol Neurosurg Psychiatry 2008;79(4):458-460

19 Bakr MS, Saleh EM. Electronystagmography: how helpful is it? J Laryngol Otol 2000;114(3):178-183

20 Ruckenstein MJ, Shepard NT. Balance function testing: a rational approach. Otolaryngol Clin North Am 2000;33(3):507-518

21 Guo Y, Zhou H. [Value of visual nystagmography in diagnosis of the patients with vertebrobasilar transient ischemic vertigo]. Lin Chung Er Bi Yan Hou Tou Jing Wai Ke Za Zhi 2007;21(14):639-642

22 Strupp M, Versino M, Brandt T. Vestibular migraine. Handb Clin Neurol 2010;97:755-771

23 Stoddart RL, Baguley DM, Beynon GJ, Chang P, Moffat DA. Magnetic resonance imaging results in patients with central electronystagmography findings. Clin Otolaryngol Allied Sci 2000;25(4): 293-297

24 Kattah JC, Talkad AV, Wang DZ, Hsieh YH, Newman-Toker DE. HINTS to diagnose stroke in the acute vestibular syndrome: threestep bedside oculomotor examination more sensitive than early MRI diffusion-weighted imaging. Stroke 2009;40(11):3504-3510

25 Dodt C, Zelihic E. [Vertigo and dizziness. Diagnostic algorithm from the perspective of emergency medicine]. Med Klin Intensivmed Notfmed 2013;108(1):41-46

26 Celebisoy N, Gökçay F, Sirin H, Biçak N. Migrainous vertigo: clinical, oculographic and posturographic findings. Cephalalgia 2008; 28(1):72-77

27 Lempert T, Neuhauser H, Daroff RB. Vertigo as a symptom of migraine. Ann N Y Acad Sci 2009;1164:242-251 